\title{
Dual-Band Antennas for Frequency-Doubler-Based Wireless Strain Sensing
}

\author{
Dora Ahbe, Stefan Beer, Student Member, IEEE, Thomas Zwick, Senior Member, IEEE, Yang Wang, and \\ Manos M. Tentzeris, Fellow, IEEE
}

\begin{abstract}
In this letter, two dual-band antennas operating at 2.9 and $5.8 \mathrm{GHz}$ are proposed and investigated in terms of the strain dependency of their resonances. They are designed to operate as strain-sensing and communicating devices at the same time in a frequency-doubling strain sensor that is interrogated wirelessly by a reader. An integration of transmit and receive antennas into one dual-band structure offers a compact sensor solution. The antennas' resonance frequencies shift if strain is applied, which in the case of the proposed antennas can be used to characterize the strain not only in terms of amplitude, but also direction. A very small size is achieved for an optimized one-feed design, which leads to high strain sensitivity values along one axis. A novel two-feed concept is designed to ease integration with the frequency doubler and detect strain in two directions with equal sensitivity. Return loss and radiation patterns of both designs are presented. Due to the wide beamwidths of both antennas, interrogation is possible from a wide range of angles.
\end{abstract}

Index Terms-Frequency doubler, microstrip antennas, multifrequency antennas, strain measurement, structural health monitoring (SHM), wireless sensors.

\section{INTRODUCTION}

$\mathbf{S}$ TRUCTURAL health monitoring (SHM) is the process of continuous surveillance and damage detection on buildings, aircrafts, machines, or other civil structures. A system of sensors ("smart skin") allows the detection and characterization of damages that could have a significant effect on the operational capability of the structure. Ideally, this will provide warnings, prevent complete failure, and facilitate countermeasures.

SHM sensors detect various parameters such as temperature, humidity, or strain. The characterization of strain gives information on cracks, deformations, or vibrations on the structure. It is therefore an essential parameter for the conclusion on operability.

Manuscript received October 14, 2011; revised November 25, 2011; accepted January 27, 2012. Date of publication February 14, 2012; date of current version March 19, 2012. This work was supported by the Federal Highway Administration and the NSF under Grant ECS-0801798.

D. Ahbe was with the Georgia Institute of Technology (Georgia Tech), Atlanta, GA 30332 USA. She is with the Institut fuer Hochfrequenztechnik und Elektronik (IHE), Karlsruhe Institute of Technology, 76131 Karlsruhe, Germany (e-mail: dora.ahbe@student.kit.edu).

S. Beer and T. Zwick are with the Institut fuer Hochfrequenztechnik und Elektronik (IHE), Karlsruhe Institute of Technology, 76131 Karlsruhe, Germany.

Y. Wang is with the Department of Civil and Environmental Engineering, Georgia Institute of Technology (Georgia Tech), Atlanta, GA 30332 USA.

M. M. Tentzeris is with the Department of Electrical and Computer Engineering, Georgia Institute of Technology (Georgia Tech), Atlanta, GA 30332 USA.

Color versions of one or more of the figures in this letter are available online at http://ieeexplore.ieee.org.

Digital Object Identifier 10.1109/LAWP.2012.2188014

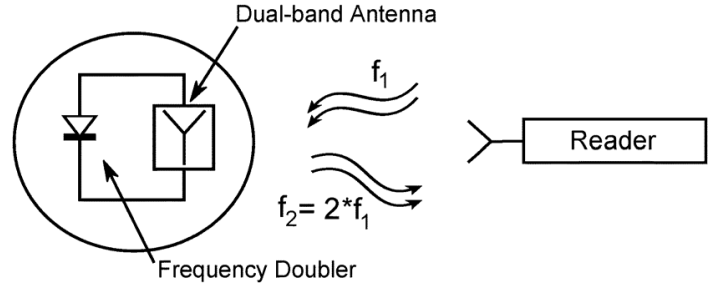

Fig. 1. Interrogation of dual-band sensor antenna with frequency doubler.

The most commonly used sensors for strain monitoring today are piezoresistive strain gauges. Extensive wiring is required to connect these sensors to a base station, which gathers the sensed data. This adds a significant amount of complexity and error sources to the system if, for example, a large bridge has to be equipped [1]. The solution can be the use of wireless sensors, which can be attached to the monitored structure and interrogated wirelessly by a reader. A high level of compactness can be achieved if the antenna at the sensor that is used for communication purposes with the reader device serves as the sensing part at the same time [2]. If the antenna is tightly attached to known weak points of the monitored object, the strain on the structure transfers to the antenna, which then changes its dimensions and hereby its resonance frequency [2], [3]. By monitoring the shift of the antenna's resonance frequency, the applied strain can thus be evaluated.

The principle of a harmonic radar can be used to assign separate frequency bands to the interrogation and sensor response signal for different function discrimination as well as for the elimination of interfering ambient clutter scattering [4]. In [5], two separate antennas are used for the operation in the two frequency bands. However, to ensure strain homogeneity over the sensor parts and make the sensor more compact, a dual-band antenna is favored. For a doubler-based wireless strain sensor concept as illustrated in Fig. 1, feasible dual-band antenna concepts have to be found that provide a high sensitivity to strain, make strain detection in different directions possible, offer integration possibilities with the frequency-doubling device, and facilitate interrogation by a reader. In this letter, a one-feed antenna introduced by [6] and a new two-feed antenna design are proposed and investigated on their feasibility as a strain sensor in the named system.

\section{ANTENNA CONCEPTS}

\section{A. Effect of Strain on a Microstrip Antenna}

The resonant frequency of a rectangular microstrip antenna that consists of a metallic layer on a substrate material with a ground plane on the other side is mainly dependent on the physical length $L$ of the patch. Fringing field effects on the patch 
edges introduce an imaginary line extension $\Delta L_{\text {ext }}$, which adds up to the physical length and is linearly dependent on the substrate thickness $h$ [7]. The antenna's resonance frequency is then [3]

$$
f_{\text {res }}=\underbrace{\frac{c_{0}}{2 \sqrt{\epsilon_{\mathrm{r}, \mathrm{eff}}}}}_{k_{1}} \frac{1}{L+2 \Delta L_{\mathrm{ext}}}=\frac{k_{1}}{L+k_{2} h}
$$

where $k_{1}$ and $k_{2}$ are constants.

Strain is defined as the deformation of a body $\Delta L$ in relation to its initial size $L_{0}$ due to the application of force. For one dimension, strain is given by [8]

$$
\varepsilon=\frac{\Delta L}{L_{0}} .
$$

Strain is here given in $\mu \varepsilon$ (microstrain), with $1 \mu \varepsilon$ corresponding to $0.0001 \%$ of elongation.

If a strain $\varepsilon_{l}$ is applied on an antenna parallel to the length $L_{0}$, which determines the antennas resonance, its dimensions are affected with respect to Poisson's effect [8] as follows:

$$
\begin{aligned}
L & =L_{0}\left(1+\varepsilon_{l}\right) \\
W & =W_{0}\left(1-\nu_{\mathrm{p}} \varepsilon_{l}\right) \\
h & =h_{0}\left(1-\nu_{\mathrm{s}} \varepsilon_{l}\right)
\end{aligned}
$$

where $W$ is the width of the patch, $\nu_{\mathrm{p}}$ and $\nu_{\mathrm{s}}$ are the Poisson's ratios of the metal layer and the substrate material, respectively, and the index 0 indicates the initial body dimensions. This deformation then leads to a frequency downshift, the new resonance according to (1) being [3]

$$
f_{\mathrm{res}}\left(\varepsilon_{L}\right)=\frac{k_{1}}{L_{0}\left(1+\varepsilon_{l}\right)+k_{2} h_{0}\left(1-\nu_{\mathrm{s}} \varepsilon_{l}\right)} .
$$

This leads to the idealized relationship between resonance frequency and strain (it is assumed that $\nu_{\mathrm{s}}=\nu_{\mathrm{p}}=\nu$ )

$$
\varepsilon_{L}=-\frac{L_{0}+\nu k_{2} h_{0}}{L_{0}+k_{2} h_{0}} \frac{\Delta f_{\text {res }}}{f_{\text {res } 0}}=K \frac{\Delta f_{\text {res }}}{f_{\text {res } 0}}
$$

with $\Delta f_{\text {res }}$ being the shift of the resonance frequency. The shift in resonance frequency is thus linearly dependent on the applied strain level. The strain sensitivity can be defined as $\Delta f_{\text {res }} / \varepsilon_{L}$ and is given in $\mathrm{kHz} / \mu \varepsilon$.

If strain is applied in cross direction to the electrical length of an antenna, the length of the patch decreases due to Poisson's effect. This leads to an upshift in resonance frequency. The direction of applied strain can therefore be detected by the prefix of the frequency shift. In case of a dual-band antenna, the two resonances can shift in equal or opposing directions.

\section{B. One-Feed Antenna}

A rectangular patch with slots along its width was introduced in [6]. Dimension adjustments were made as can be seen in Fig. 2 to match the two resonances to the desired frequency bands and increase radiation efficiency at the first resonance. For $f_{1}$, the entire physical length of the antenna resonates. Due to the inductive loading of the slots, an antenna size of only $47 \%$ of the size of a rectangular $\lambda / 2$-patch resonating at $2.9 \mathrm{GHz}$ was

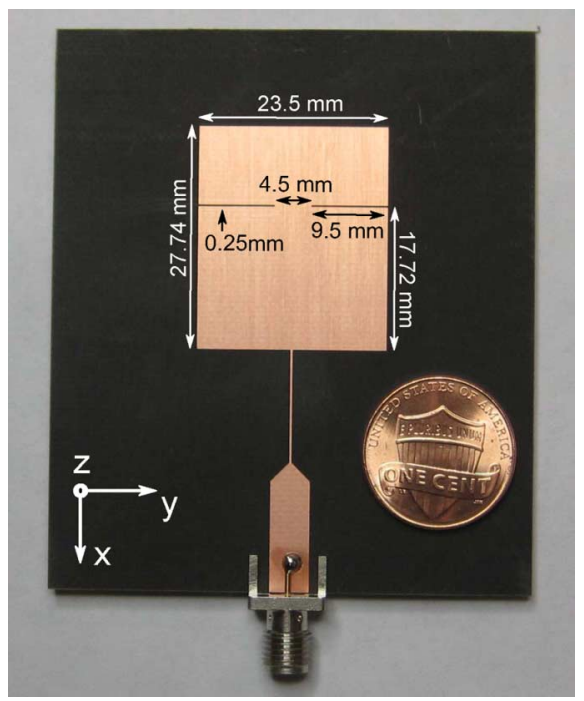

Fig. 2. One-feed antenna.

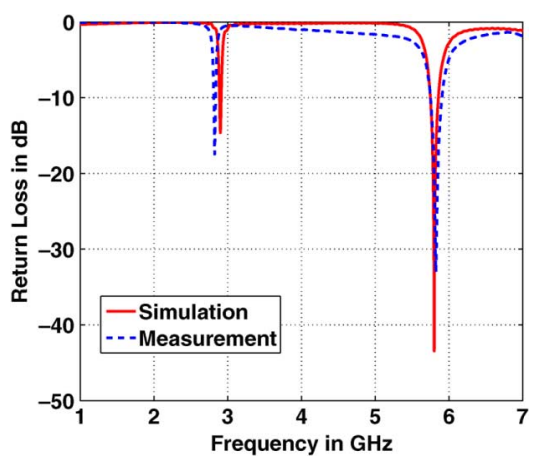

Fig. 3. Simulated and measured return loss for the one-feed antenna.

achieved. For higher frequencies, the slots function as a filter for the upper antenna part. Only the lower part of the antenna between the slots and the bottom edge resonates, creating the second resonance at $5.8 \mathrm{GHz}$

The antenna was fabricated on Rogers RT/duroid 5880 substrate material with $1.575 \mathrm{~mm}$ thickness. Simulated and measured return loss can be seen in Fig. 3. In order to increase the radiation efficiency at the first resonance frequency, which is limited due to the introduction of the slots, the width and length of the slots were optimized. It was found that narrowing the width of the slots and decreasing their length increases efficiency at $f_{1}$. However, a notch is introduced in the radiation pattern at $f_{2}$ if the length of the slots is decreased due to the worsening performance of the slots as filter. A compromise was found with the notch being above $-3 \mathrm{~dB}$ in depth. The simulated and measured radiation patterns are displayed in Figs. 4 and 5, respectively. Peak gain values of $5.1 \mathrm{dBi}$ at $f_{1}$ and $8.4 \mathrm{dBi}$ at $f_{2}$ were measured with efficiencies reaching $62.7 \%$ and $94.1 \%$ for the two resonances. With half-power beamwidths (HPBWs) of $74.5^{\circ}$ and $87.1^{\circ}$ for the two planes at $f_{1}$ and $126.8^{\circ}$ and $86.4^{\circ}$ for the two planes at $f_{2}$, reader positioning is possible over a wide range of angles over the sensing antenna. The advantage of this design is its miniaturization. However, for integration with a doubler-based system, a diplexer or a power divider has 


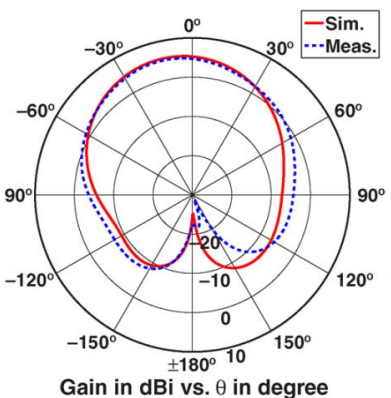

(a)

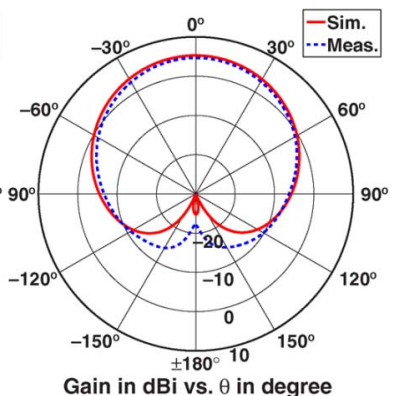

(b)
Fig. 4. Simulated and measured gain of the one-feed antenna at $2.9 \mathrm{GHz}$. (a) E-plane $\left(\psi=0^{\circ}\right)$. (b) H-plane $\left(\psi=90^{\circ}\right)$.

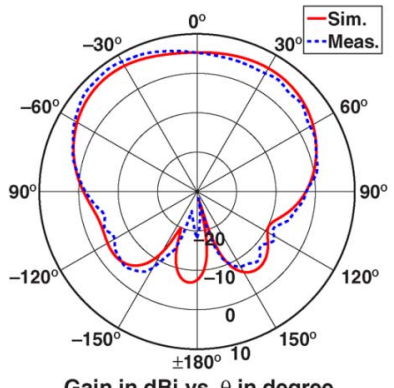

Gain in dBi vs. $\theta$ in degree

(a)

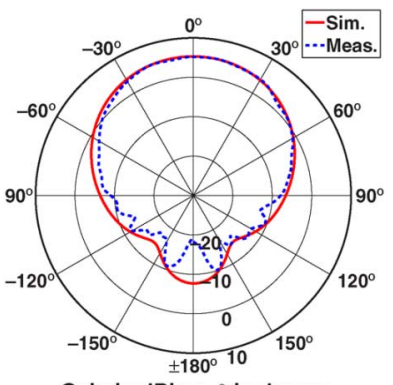

Gain in dBi vs. $\theta$ in degree

(b)
Fig. 5. Simulated and measured gain of the one-feed antenna at $5.8 \mathrm{GHz}$. (a) E-plane $\left(\psi=0^{\circ}\right)$. (b) H-plane $\left(\psi=90^{\circ}\right)$.

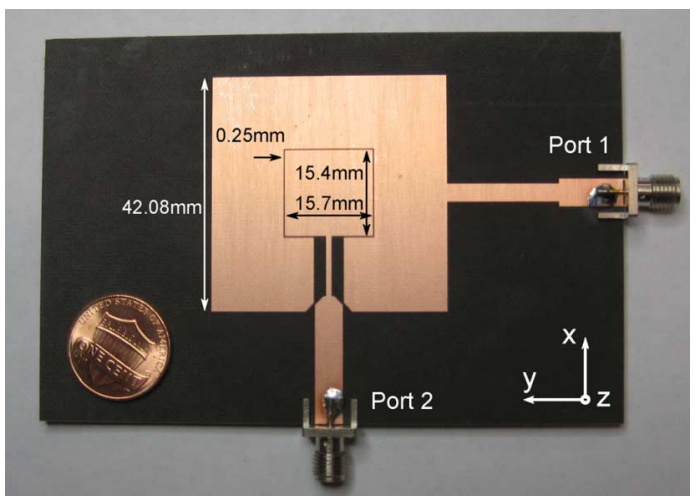

Fig. 6. Two-feed antenna.

to be employed, which introduces losses. An easier integration is possible with a two-feed antenna.

\section{Two-Feed Antenna}

A rectangular patch antenna that is surrounded by an open loop fed at a relative angle of $90^{\circ}$ was designed. The structure is an integration of two rectangular patches into one dual-band antenna with separate ports. Each port is associated with one resonance: Port 1 with $2.9 \mathrm{GHz}$; Port 2 with $5.8 \mathrm{GHz}$. The fabricated design can be seen in Fig. 6 .

For $f_{1}$, the antenna operates in a higher-order $\mathrm{TM}_{110}$ mode, making the resonance dependent not only on the physical length in $x$-direction, but also on the length in $y$-direction. For $f_{2}$, the inner patch operates in a basic-order mode, thus the resonance is mainly dependent upon its length in $x$-direction. The antenna operates in orthogonal polarizations for the two bands.

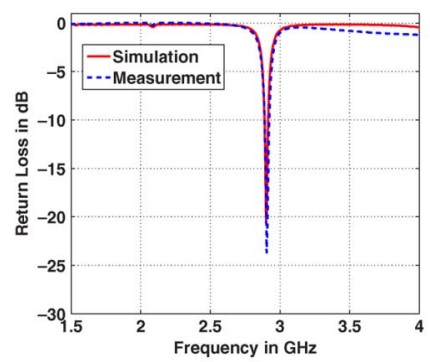

(a)

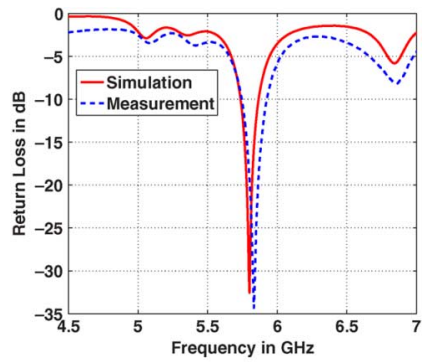

(b)
Fig. 7. Simulated and measured return loss of the two-feed antenna. (a) Port 1. (b) Port 2.

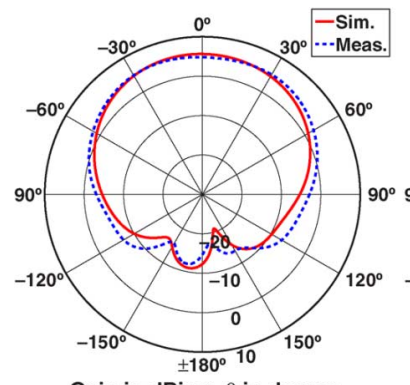

Gain in dBi vs. $\theta$ in degree

(a)

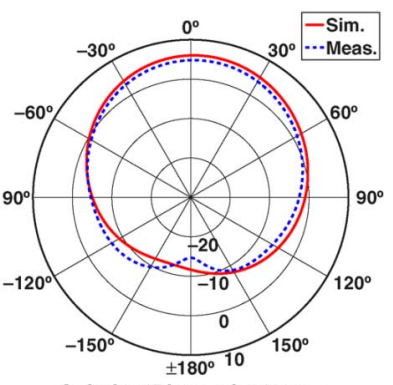

Gain in dBi vs. $\theta$ in degree

(b)
Fig. 8. Simulated and measured gain of the two-feed antenna at $2.9 \mathrm{GHz}$. (a) E-plane $\left(\psi=0^{\circ}\right)$. (b) H-plane $\left(\psi=90^{\circ}\right)$.

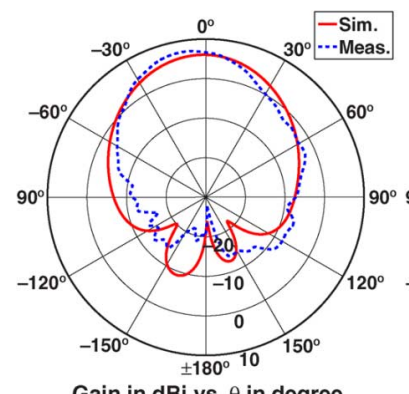

Gain in dBi vs. $\theta$ in degree

(a)

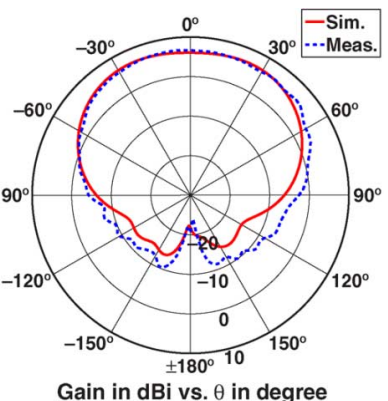

(b)
Fig. 9. Simulated and measured gain of the two-feed antenna at $5.8 \mathrm{GHz}$. (a) E-plane $\left(\psi=0^{\circ}\right)$. (b) H-plane $\left(\psi=90^{\circ}\right)$.

Simulated and measured return loss for both ports can be seen in Fig. 7. Cross coupling between the two ports was measured to be below $-15 \mathrm{~dB}$. Both ports were matched to $50 \Omega$ with the aid of $\lambda / 4$-transformers for measurement purposes. In the integrated system, they will have to be replaced by circuits matching the antenna ports to the doubler impedances.

The simulated and measured radiation patterns for both resonances are displayed in Figs. 8 and 9. They resemble patterns from a regular rectangular patch with HPBWs of $92.2^{\circ}$ and $99.1^{\circ}$ for the two planes at $f_{1}$ and $60^{\circ}$ and $109.2^{\circ}$ for the two planes at $f_{2}$. With no sidelobes, this again facilitates reader interrogation and positioning over the antenna. Gain values of $5.1 \mathrm{dBi}$ at $f_{1}$ and $7.2 \mathrm{dBi}$ at $f_{2}$ were measured with efficiencies of $85.8 \%$ and $92 \%$ for the two frequencies.

\section{Strain SEnsitivity MEASUREMEnts}

To measure the effect of strain on the antenna, the setup displayed in Fig. 10 was used. The antenna is tightly glued onto a 


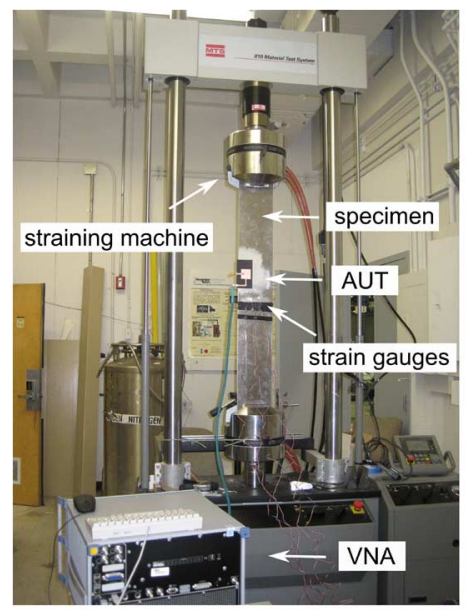

(a)

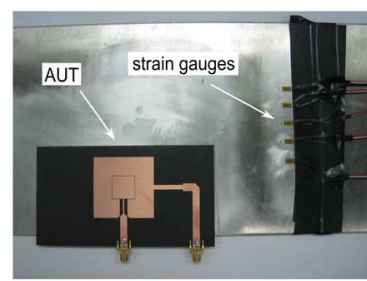

(b)
Fig. 10. Setup for strain sensitivity measurement. (a) Setup. (b) Antenna on specimen.

TABLE I

Measured Strain Sensitivities For Proposed Antennas

\begin{tabular}{|l|c|c|c|}
\hline Measured strain sensitivity in & & x-direction & y-direction \\
\hline \hline One-feed antenna & $f_{1}$ & $-2.2 \mathrm{kHz} / \mu \varepsilon$ & $0.068 \mathrm{kHz} / \mu \varepsilon$ \\
\hline & $f_{2}$ & $-3.1 \mathrm{kHz} / \mu \varepsilon$ & $0.62 \mathrm{kHz} / \mu \varepsilon$ \\
\hline Two-feed antenna & $f_{1}$ & $-0.6 \mathrm{kHz} / \mu \varepsilon$ & $-0.73 \mathrm{kHz} / \mu \varepsilon$ \\
\hline & $f_{2}$ & $-0.6 \mathrm{kHz} / \mu \varepsilon$ & $0.59 \mathrm{kHz} / \mu \varepsilon$ \\
\hline
\end{tabular}

metallic specimen, which is then clamped into a straining machine. The machine pulls on one side of the specimen to create the desired strain level on the antenna. The antenna is connected to a vector network analyzer (VNA), which records the return loss for every step of applied strain with a measurement range of $0-2500 \mu \varepsilon$. The exact strain level is detected by strain gauges that are placed on top of the specimen. The weakening of strain due to the thickness of the substrate is not taken into account by this measurement and has to be added for precise results. The antennas' transmission lines had to be extended, as can be seen in Fig. 10(b), for some measurements to make SMA connection on the side of the specimen possible.

The resonance of the antenna is extracted from the measured return loss for every step of applied strain. From these values, an average strain sensitivity of the resonance is calculated. The measured strain sensitivities are shown in Table I. The effect of strain on the antenna was also simulated. Measurement and simulation results match well for both antennas. For the one-feed antenna, high strain sensitivity values of $-2.2 \mathrm{kHz} / \mu \varepsilon$ at $f_{1}$ and $-3.1 \mathrm{kHz} / \mu \varepsilon$ at $f_{2}$ are achieved for strain along the length of the structure due to its miniaturization. The antennas' resonances shift down for this direction. If strain is applied along the width of the antenna, the resonances shift up slightly due to the Poisson's effect on the structure. The values are, however, significantly lower than for strain along the length, which means higher levels of strain are needed in order to detect the strain. Due to the different shifting directions, information on amplitude as well as direction can be extracted from the resonance shifts if the detection is limited to positive strains.

For the two-feed antenna, similar sensitivity levels were measured for both directions of applied strain. For strain in $x$-direction, both resonances shift down with $-0.6 \mathrm{kHz} / \mu \varepsilon$.
For the second resonance frequency, however, preliminary simulation results show a significantly higher sensititvity of $-3.4 \mathrm{kHz} / \mu \varepsilon$. This deviation can be explained by the influence of the extended transmission line, which had to be added to Port 1 for this measurement. This assumption was verified by an adjusted simulation.

For strain in $y$-direction, a similar sensitivity was found for $f_{1}$ compared to strain in cross direction. This is due to the operating higher-order mode on the antenna, which makes the resonance dependent on length changes along both directions. For $f_{2}$, the resonance shifts up due to Poisson's effect with $0.59 \mathrm{kHz} / \mu \varepsilon$. Since the resonances are shifting into opposing directions for strain along the $y$-axis, but in the same direction for strain along the $x$-axis, directional information can again be extracted from the shifts of the antenna resonances next to the evaluation on amplitude.

\section{CONCLUSION}

Two feasible dual-band antenna concepts for a doubler-based wireless strain sensor were proposed. A known one-feed antenna structure was optimized for the given application and a novel two-feed antenna design was introduced. The compactness of a dual-band design instead of two separate antennas ensures a certain strain homogeneity over the sensor part. Return loss, radiation pattern, and strain sensitivity were measured for both designs to verify simulation results. Both antennas are able to detect strain in terms of amplitude and direction. For the one-feed antenna, a significant size reduction compared to a rectangular patch was achieved, which leads to high strain sensitivity values along the antenna length at the expense of significant losses when integrated with frequency-doubler-based sensors. Simple low-loss integration is possible with the two-feed antenna. In addition, strain detection in two directions is possible with equal sensitivities with this design.

For higher absolute sensitivity values, the antennas can be operated in higher frequency bands after dimension adjustments.

\section{ACKNOWLEDGMENT}

The authors would like to thank K. Rutkowski from the Satimo Microwave Vision Group for measuring efficiency and radiation patterns of the proposed antennas.

\section{REFERENCES}

[1] T. Wu, "Antenna integration for wireless and sensing applications," Ph.D. dissertation, Georgia Institute of Technology, Atlanta, GA, 2011.

[2] Z. Salmani, Y. Xie, G. Zheng, H. Zhang, and H. Zhang, “Application of antenna in strain measurement," in Proc. iWAT, Mar. 2011, pp. 336-339.

[3] U. Tata, H. Huang, R. Carter, and J. Chiao, "Exploiting a patch antenna for strain measurements," Meas. Sci. Technol., vol. 20, no. 1, p. 015201 , Jan. 2009.

[4] G. Charvat, E. Rothwell, and L. Kempel, "Harmonic radar tag measurement and characterization," in Proc. IEEE Antennas Propag. Soc. Int. Symp., Jun. 2003, vol. 2, pp. 696-699.

[5] S. Presas, T. Weller, S. Silverman, and M. Rakijas, "High efficiency diode doubler with conjugate-matched antennas," in Proc. Eur. Microw. Conf., Oct. 2007, pp. 250-253.

[6] Y. Mahe, L. Desclos, R. Moreau, and S. Toutain, "Miniaturized patch antenna for dual-frequency operation," in Proc. 31st Eur. Microw. Conf., Sep. 2001, pp. 1-4.

[7] C. Balanis, Antenna Theory. Hoboken, NJ: Wiley, 2005.

[8] D. Meschede, Gerthsen Physik. Berlin, Germany: Springer-Verlag, 2010. 